\title{
Risk factors for cardiovascular mortality in patients with systemic lupus erythematosus, a prospective cohort study
}

Johanna T Gustafsson ${ }^{1 *}$, Julia F Simard ${ }^{2}$, Iva Gunnarsson ${ }^{1}$, Kerstin Elvin ${ }^{3}$, Ingrid E Lundberg ${ }^{1}$, Lars-Olof Hansson ${ }^{4}$, Anders Larsson ${ }^{5}$ and Elisabet Svenungsson ${ }^{1}$

\begin{abstract}
Introduction: Systemic lupus erythematosus (SLE) is a chronic autoimmune disease. Cardiovascular disease (CVD) is common and a major cause of mortality. Studies on cardiovascular morbidity are abundant, whereas mortality studies focusing on cardiovascular outcomes are scarce. The aim of this study was to investigate causes of death and baseline predictors of overall (OM), non-vascular (N-VM), and specifically cardiovascular (CVM) mortality in SLE, and to evaluate systematic coronary risk evaluation (SCORE).

Methods: 208 SLE patients were included 1995-1999 and followed up after 12 years. Clinical evaluation, CVD risk factors, and biomarkers were recorded at inclusion. Death certificates and autopsy protocols were collected. Causes of death were divided into CVM (ischemic vascular and general atherosclerotic diseases), N-VM and death due to pulmonary hypertension. Predictors of mortality were investigated using multivariable Cox regression. SCORE and standardized mortality ratio (SMR) were calculated.

Results: During follow-up 42 patients died at mean age of 62 years. SMR 2.4 (Cl 1.7-3.0). 48\% of deaths were caused by CVM. SCORE underestimated CVM but not to a significant level. Age, high cystatin C levels and established arterial disease were the strongest predictors for all- cause mortality. After adjusting for these in multivariable analyses, only smoking among traditional risk factors, and high soluble vascular cell adhesion molecule-1 (sVCAM-1), high sensitivity C-reactive protein (hsCRP), anti-beta2 glycoprotein-1 (abeta2GP1) and any antiphospholipid antibody (aPL) among biomarkers, remained predictive of CVM.

Conclusion: With the exception of smoking, traditional risk factors do not capture the main underlying risk factors for CVM in SLE. Rather, cystatin C levels, inflammatory and endothelial markers, and antiphospholipid antibodies (aPL) differentiate patients with favorable versus severe cardiovascular prognosis. Our results suggest that these new biomarkers are useful in evaluating the future risk of cardiovascular mortality in SLE patients.
\end{abstract}

\section{Introduction}

Systemic lupus erythematosus (SLE) is an autoimmune rheumatic disease predominately affecting women (90\%). Clinical manifestations are systemic, affecting organs including skin, joints, kidneys and the vascular system.

Cardiovascular disease (CVD) is a well studied co-morbidity of SLE with many remaining questions to be answered. Both subclinical CVD, measured as

\footnotetext{
* Correspondence: johanna.gustafsson@karolinska.se

'Rheumatology Unit, Department of Medicine, Karolinska University Hospital,

Solna, Karolinska Institutet, SE-171 76 Stockholm, Sweden

Full list of author information is available at the end of the article
}

atherosclerosis, and clinical events have been subjects for investigation. Studies have focused on different aspects of the disease to find associations with, and to characterize, SLE-related CVD. For example, CVD in SLE has been associated with clinical manifestations, disease activity and damage, traditional and nontraditional riskfactors, and demographic factors [1-4]. Risk factors for cardiovascular mortality (CVM) in SLE on the other hand, have not yet been well studied.

In the 1950s, the estimated 5-year survival was less than 50\% [5], but recent studies report 5-year survival of over $90 \%[6,7]$. Nevertheless, the mortality rate in SLE

\section{Biomed Central}


still exceeds that of the general population $[8,9]$. Death related to lupus activity and infection has decreased over time, but still contributes to mortality $[10,11]$, especially in developing countries[12,13]. However, CVM has not declined [14] in SLE. A slight increased standardized mortality ratio (SMR) due to vascular diseases has been reported [15], and death from CVD accounts for between $17 \%$ and $76 \%$ in different studies [16,17]. To date, most studies have investigated risk factors for overall mortality $(\mathrm{OM})$, sometimes with diverging results [10,12,18-21]. As CVM accounts for a growing part of mortality in SLE, it is important to identify risk factors specifically for CVM. In the general population, the systematic coronary risk evaluation (SCORE) [22] is a wellestablished tool to predict the 10-year risk of CVM based on traditional risk factors. SCORE has not previously been evaluated in SLE. Many new biomarkers that could help identify underlying molecular pathways of importance for vascular damage, such as endothelial and inflammatory markers and cystatin $\mathrm{C}$ have not been evaluated with respect to mortality in SLE.

Therefore, we described a large set of biomarkers and SCORE in a cohort of 208 SLE patients from a single center. We determined causes of death and the contribution of baseline predictors for OM, CVM and non-vascular mortality (N-VM).

\section{Materials and methods}

During the inclusion period (1995 to 1998), 208 patients with prevalent disease, who were attending the Department of Rheumatology, Karolinska University Hospital, and fulfilled four or more of the 1982 revised American College of Rheumatology criteria for classification of SLE [23] were included. Most patients (94\%) were European Caucasians. The Local Ethics Committee at Karolinska University Hospital approved the study and patients provided informed consent.

At inclusion, all data were collected in one session for each patient. A rheumatologist interviewed and examined patients according to a structured protocol. Medical history, traditional CVD risk factors (smoking, hypertension, hypercholesterolemia, diabetes) and medication were reviewed, through interviewing the patient and by studying medical records. SLE disease activity was determined using the Systemic Lupus Activity Measure (SLAM) [24] and organ damage was assessed using the Systemic Lupus International Collaborating Clinics (SLICC) damage index [25]. Laboratory examinations were performed on fasting fresh blood samples or on samples stored at $-70^{\circ} \mathrm{C}$. When stored samples were used, each analyte was assayed in one session.

Survival status was followed up in the national population registry on March 26, 2010, after a mean time of 12.3 years. Two patients were interviewed by telephone as they had moved abroad. Death certificates were collected from the Cause of Death Register of The National Board of Health and Welfare. When available, autopsy protocols were collected from the department of Pathology, Karolinska University Hospital $(\mathrm{n}=10)$, and from the Department of Forensic Medicine $(\mathrm{n}=4)$. Causes of death were based on information from death certificates, autopsy protocols and medical records. Two clinicians (JG and ES) classified all causes of death together as follows: CVM (death due to myocardial infarction, atherosclerosis, heart failure, ischemic cerebrovascular disease or sudden death), death due to pulmonary hypertension (PHT) and N-VM.

\section{Laboratory methods}

High sensitivity C-reactive protein (hsCRP), $\alpha-1$ antitrypsin, fibrinogen and serum amyloid A (SAA) were measured using BN ProSpec System (Dade Behring, Göttingen, Germany). Complement factors C3 and C4 were analyzed using IMMAGE ${ }^{\mathrm{TM}}$ and C3d using an ARRAYTM system (both instruments from Beckman Coulter, Brea, CA, USA). Apolipoprotein A1, apolipoprotein B, creatinine, high-density lipoprotein (HDL), low-density lipoprotein (LDL) and total cholesterol, triglycerides and urea were analyzed using the LX20 chemistry analyzer (Beckman Coulter, Brea, CA, USA). Homocysteine was assayed using the IMx system and Cystatin $C$ was analyzed using the Architect Ci8200 analyzer (both by Abbott Laboratories, Chicago IL, USA). ELISA was used to measure soluble vascular cell adhesion molecule (sVCAM-1) and IL-6 (DY809 and HS600, R\&D Systems, Minneapolis, MN, USA) and von Willebrand factor (vWF) (antisera from Dako, Glostrup, Denmark) was calibrated against Liatest (Diagnostica Stago, Asnières sur Seine, France). The intra-assay coefficient of variation for the ELISAs was $<7 \%$.

Antinuclear antibodies (ANA) was analyzed by immunofluorescence on HEp-2 cells (Immunoconcepts, Sacramento, CA, USA) and antibodies to Sjogrens syndrome A and B (SSA, SSB), Smith antigen (Sm) and Ribonucleoprotein (RNP) using ANA-profile ELISA (Thermo Fisher Scientific, previously Phadia, Uppsala, Sweden), Innolia immunoblot (Innogenetics, N.U. Ghent, Belgium), and immunodiffusion (Immunoconcepts, Sacramento, CA, USA). Anti-dsDNA was determined by Crithidia lucillae kinetoplast assay (Immunoconcepts, Sacramento, CA, USA). Anticardiolipin antibodies (aCL) were measured by ELISA using ethanol-fixed cardiolipin (Sigma-Aldrich, St Louis, MO, USA) and horseradish peroxidase (HRP)conjugated rabbit anti-human IgG and IgM (Dako, Glostrup, Denmark). Positivity was calibrated against Harris standard (Louisville, LAPL-GM-001, Louisville, KY, USA) [26]. Low aCL level corresponded to 10 to 20 , medium level to 20 to 80 and high level to $>80 \mathrm{GPU} / \mathrm{mPL}$ 
units. The cutoff values for positive aCL was calculated to be at least the $95^{\text {th }}$ percentile of healthy blood donors. Autoantibodies to $\beta 2$-glycoprotein 1 ( $a \beta_{2} \mathrm{GP} 1, \mathrm{IgG}$ ) were analyzed by ELISA (Orgentec Diagnostika, Mainz, Germany). Positive cutoff levels were used according to the manufacturers' descriptions. Borderline results were regarded as negative. Lupus anticoagulant (LAC) was determined using a modified dilute Russell viper venom method, (Biopool, Umea, Sweden) using Bioclot LAC.

\section{Statistics}

Patient characteristics were summarized overall and stratified by outcome using analysis of variance (ANOVA), Mann-Whitney $U$-test and $\mathrm{Chi}^{2}$ test, as appropriate. Skewed continuous variables were log transformed before use in parametric analyses. The SMR for all deaths and corresponding 95\% confidence interval (CI) assuming a Poisson distribution was calculated using age-, sex-, and calendar year-specific mortality rates for the Swedish population to estimate the expected number of deaths. Hazard ratios (HRs), it should be hazard ratios everywhere and 95\% CI for OM, CVM and N-VM were calculated in age-adjusted Cox models for baseline factors. The proportional hazards assumption was evaluated by assessing the significance of the interaction between predictors and follow-up time and the assumption was met. PHT was included in OM but otherwise excluded because of too few cases. The limited number of deaths restricted the variables in the multivariable-adjusted model to four. The two predictors most strongly associated with mortality after age adjustment (in terms of $P$-value) in the three groups (OM, CVM, N-VM) were retained in all multivariable analyses. Thereafter, each baseline variable was considered separately in multivariable models.

To evaluate the different multivariable models predicting CVM in terms of identifying the best model, and to compare that model with SCORE, Akaike information criterion (AIC) values were compared using logistic regression. To consider the possibility of effect modification by sex, we stratified by sex; we restricted the sensitivity analysis to the female subset. Male patients were not considered due to small sample size. Possible effect modification on cystatin $C$ by steroid treatment was evaluated by stratifying by steroid treatment. To account for the possibility that subclinical or unregistered nephritis could affect the cystatin $C$ results, we also stratified by history of nephritis.

SCORE [22] was calculated using the Swedish Heartscore. Baseline data on age, smoking, sex, systolic blood pressure and cholesterol were incorporated into the webbased formula [27]. The 10-year risk of CVM was calculated for patients between 40 to 65 years of age, according to the SCORE protocol. The estimated number of CVMs was compared to the observed number using Fisher's exact analysis.
Multiple imputation was used for missing data. For dichotomous variables the models were re-run assuming each possible value and the results were compared. For continuous predictors, three imputed values were used: the minimum, the mean, and the maximum value. Descriptive statistics and regression analyses were done using JMP software (SAS Institute, Carey, North Carolina, USA) and SAS 9.2 was used for SMR calculations. A $P$-value $\leq 0.05$ was considered statistically significant.

\section{Results}

All patients were followed up. Forty-two patients (20\%) died, 36 women and 6 men, at a mean age of 62 years (women $61 \pm$ SD 14, men $64 \pm 19$ years). More deaths were observed than expected $(\mathrm{SMR}=2.4,95 \% \mathrm{CI} 1.7$ to 3.0). CVM was the predominant cause of death $(n=20$, 48\%) (Table 1).

At inclusion, 124 patients were between 40 and 65 years of age. In this group, we observed nine cardiovascular deaths within 10 years. SCORE only predicted four deaths and the difference was not statistically significant (odds ratio, OR 2.3, 95\% CI 0.7 to $7.8, P=0.25$ ).

Several parameters measured at baseline differed between deceased and surviving patients (Table 2) and persisted after age adjustment for all outcomes. Established arterial disease and Cystatin $\mathrm{C}$ were the strongest risk factors in all groups (Table 3 ) and were retained in the remaining analyses (Table 4). OM was predicted by several inflammatory parameters, sVCAM-1 and SLICC $>1$.

Smoking was the only traditional risk factor predicting CVM. sVCAM-1, hsCRP, a $\beta_{2}$ GP1, any aPL at medium titer, and baseline warfarin treatment also predicted CVM. $\mathrm{N}-\mathrm{VM}$ was positively associated with markers of systemic inflammation and SLICC >1, while SSB autoantibodies were inversely associated (Table 4). Results were similar among women, with a few exceptions (see Table 5). The best multivariable model predicting CVM included age, established arterial disease, cystatin $\mathrm{C}$ and smoking (AICc value 77). Yes, the same thing All six multivariable models predicting CVM (AICc values ranging from 77 to 85 ) performed better than SCORE (AICc value 122).

Stratification by steroid treatment did not influence the impact of cystatin $C$ on mortality. Among those without history of nephritis ( $\mathrm{n}=135$ of whom 25 died cystatin $\mathrm{C}$ adjusted for age remained significant; $P=$ 0.009 , RR 4.6 (95\% CI 1.5 to 14.5 ). For patients with history of nephritis ( $\mathrm{n}=73$ of whom 17 died), the results for cystatin $C$ were similar; $P=0.001$, RR 4.4 (95\% CI 1.8 to 10.7$)$.

Results were comparable under the numerous imputed scenarios with the exception of cyclophosphamide, where a large proportion of missingness was observed among deceased patients, and alternative imputed scenarios 
Table 1 Causes of death in deceased patients $(n=42)$

\begin{tabular}{|c|c|c|c|c|}
\hline Primary cause of death & $\begin{array}{l}\text { Number of } \\
\text { patients }\end{array}$ & $\begin{array}{l}\text { Age at death, } \\
\text { years }\end{array}$ & $\begin{array}{l}\text { Age at SLE diagnosis, } \\
\text { years }\end{array}$ & $\begin{array}{l}\text { Disease duration at baseline, } \\
\text { years }\end{array}$ \\
\hline Cardiovascular mortality & $20(48 \%)$ & $69( \pm 11)$ & $43( \pm 18)$ & $19( \pm 14)$ \\
\hline Myocardial infarction & 10 & & & \\
\hline Congestive.heart failure & 7 & & & \\
\hline CVLcerebrovascular lesion & 1 & & & \\
\hline Atherosclerosis & 1 & & & \\
\hline Sudden death & 1 & & & \\
\hline Non-vascular mortality & 18 & $57( \pm 13)$ & $36( \pm 13)$ & $15( \pm 10)$ \\
\hline Infection & $5(12 \%)$ & $46( \pm 12)$ & $20( \pm 6.8)$ & $16( \pm 10)$ \\
\hline Sepsis & 3 & & & \\
\hline Pneumonia & 1 & & & \\
\hline Perforation of esophagus & 1 & & & \\
\hline Bleeding & $4(10 \%)$ & $56( \pm 16)$ & $32( \pm 13)$ & $20( \pm 9)$ \\
\hline Gastrointestinal bleeding & 3 & & & \\
\hline Unknown & 1 & & & \\
\hline Malignancy & $4(10 \%)$ & $62( \pm 6)$ & $37( \pm 10)$ & $18( \pm 9)$ \\
\hline Lung cancer & 2 & & & \\
\hline Colorectal cancer & 1 & & & \\
\hline Squamous cell cancer & 1 & & & \\
\hline Suicide & $2(5 \%)$ & $64( \pm 3)$ & $51( \pm 19)$ & $11( \pm 13)$ \\
\hline $\begin{array}{l}\text { Pulmonary disease } \\
\text { (pneumothorax) }\end{array}$ & $1(2 \%)$ & 54 & 46 & 6 \\
\hline Renal failure & $1(2 \%)$ & 85 & 73 & 2 \\
\hline Hepatic failure (cirrhosis) & $1(2 \%)$ & 67 & 54 & 2 \\
\hline Pulmonary hypertension & $4(10 \%)$ & $41( \pm 16)$ & $29( \pm 13)$ & $10( \pm 9)$ \\
\hline Total/Average & 42 & $62( \pm 14)$ & $33( \pm 13)$ & $13( \pm 10)$ \\
\hline
\end{tabular}

Age is given as mean \pm SD.

yielded different results. As these were considered unreliable, they were removed from consideration.

\section{Discussion}

To our knowledge, this is the first study to prospectively examine risk factors specifically for CVM, which accounted for almost $50 \%$ of deaths in our cohort. Additionally, $10 \%$ of patients died from PHT. CVM and N-VM shared many risk factors. Established arterial disease, Cystatin $\mathrm{C}$ and inflammatory markers were strong predictors for both, but s-VCAM-1, a marker of endothelial cell activation, was only associated with CVM. Also, $\mathrm{a} \beta_{2} \mathrm{GP} 1$ and any aPL at medium titer predicted CVM. SCORE underestimated the risk for CVM, but the results were not statistically significant. Consistent with recently published work [9,15], SMR was 2.4. Our survival rate of $80 \%$ after a mean follow-up of approximately 12 years is generally consistent with previous findings, which range from $76 \%$ to $92 \%$ survival after 10 years $[28,29]$. Our results confirm $[6,17,30]$ that composite damage (SLICC $>1[25]$ ) predicted OM, but our focus was to analyze the impact of different organ manifestations and immunological profile on mortality.
High levels of cystatin $\mathrm{C}$ and low estimated glomerular filtration rate (eGFR) based on cystatin C [31] emerged as strong predictors for all outcomes. These associations were independent of inflammatory and endothelial biomarkers and were not modified by steroid treatment at baseline. Creatinine and eGRF, calculated using the Modification of Diet in Renal Disease (MDRD) formula [32] did not predict mortality.

Renal disease in lupus is associated with poor prognosis $[10,20,33]$. Cystatin C has been proposed as a more reliable biomarker for renal function than creatinine as it rises with smaller reductions in GFR [34], and is less influenced by age, sex, muscle mass and diet [35]. Nevertheless, cystatin $C$ levels may be affected by glucocorticoid use [36] and inflammation [37], both often present in SLE. Cystatin C has furthermore emerged as a marker of CVD risk [38], CVM and N-VM in subjects with normal eGFR [39]. The fact that the nephritis manifestation in our study only influenced CVM to a modest degree, while cystatin $C$ predicted mortality significantly both in patients with and without reported history of nephritis, further emphasizes the importance of cystatin $C$ as a new useful biomarker. Our results demonstrate that cystatin 
Table 2 Baseline characteristics of patients with SLE $(n=208)$

\begin{tabular}{|c|c|c|c|c|}
\hline & All patients $n=208$ & $\begin{array}{l}\text { Deceased patients } \\
\quad \mathrm{n}=42\end{array}$ & Surviving patients $\mathrm{n}=166$ & $P$ \\
\hline \multicolumn{5}{|l|}{ Traditional risk factors } \\
\hline Age & $47(35-54)$ & $57(48-65)$ & $45(31-52)$ & $<0.0001$ \\
\hline Male gender & $11 \%$ & $14 \%$ & $10 \%$ & \\
\hline Smoking & $24 \%$ & $26 \%$ & $22 \%$ & \\
\hline Hypertension & $34 \%$ & $62 \%$ & $28 \%$ & $<0.0001$ \\
\hline Systolic blood pressure, mmHg & $125(115-140)$ & $140(123-148)$ & $120(110-140)$ & 0.001 \\
\hline Hypercholesterolemia & $44 \%$ & $55 \%$ & $41 \%$ & \\
\hline Total cholesterol, (mmol/l) & $5.1(4.3-6.0)$ & $5.6(4.7-6.7)$ & $5.0(4.3-5.9)$ & 0.04 \\
\hline Low density lipoprotein mmol/l & $2.9(2.3-3.6)$ & $3.3(2.2-3.9)$ & $2.8(2.3-3.6)$ & \\
\hline High density lipoprotein, mmol/l & $1.4(1.1-1.7)$ & $1.4(1.1-1.9)$ & $1.4(1.1-1.7)$ & \\
\hline Triglycerides, $\mathrm{mmol} / /^{*}$ & $1.3(1.0-2.0)$ & $1.7(1.2-2.5)$ & $1.2(0.9-1.9)$ & 0.004 \\
\hline ApolipoproteinB/Apolipoprotein $A^{*} \#$ & $0.5(0.3-0.6)$ & $0.5(0.3-0.8)$ & $0.5(0.3-0.6)$ & \\
\hline Diabetes & $3 \%$ & $10 \%$ & $2 \%$ & 0.03 \\
\hline Established arterial disease & $13 \%$ & $40 \%$ & $5 \%$ & $<0.0001$ \\
\hline SCORE & $3.2 \%( \pm 2.8)$ & $4.4 \%( \pm 2.4)$ & $2.7 \%( \pm 2.4)$ & 0.005 \\
\hline \multicolumn{5}{|l|}{ Lupus manifestations (ever present)§ } \\
\hline Age at disease onset, years & $30(22-40)$ & $35(25-52)$ & $28(21-40)$ & 0.0002 \\
\hline Disease duration, years & $12(5-20)$ & $13.5(6-26)$ & $12(5-19)$ & 0.008 \\
\hline Malar rash & $56 \%$ & $50 \%$ & $58 \%$ & \\
\hline Discoid rash & $20 \%$ & $12 \%$ & $21 \%$ & \\
\hline Photosensitivity & $71 \%$ & $60 \%$ & $73 \%$ & \\
\hline Oral ulcers & $28 \%$ & $28 \%$ & $26 \%$ & \\
\hline Arthritis & $86 \%$ & $86 \%$ & $86 \%$ & \\
\hline Pleuritis & $40 \%$ & $52 \%$ & $37 \%$ & \\
\hline Pericarditis & $18 \%$ & $17 \%$ & $19 \%$ & \\
\hline Nephritis & $35 \%$ & $40 \%$ & $34 \%$ & \\
\hline Neurological disorder & $15 \%$ & $29 \%$ & $12 \%$ & 0.009 \\
\hline Leucopenia & $51 \%$ & $37 \%$ & $55 \%$ & 0.04 \\
\hline Thrombocytopenia & $22 \%$ & $30 \%$ & $20 \%$ & \\
\hline Previous venous occlusion & $11 \%$ & $17 \%$ & $9 \%$ & \\
\hline SLICC > $1[25]$ & $59 \%$ & $93 \%$ & $50 \%$ & $<0.0001$ \\
\hline SLAM > $6[24]$ & $59 \%$ & $73 \%$ & $55 \%$ & 0.03 \\
\hline \multicolumn{5}{|l|}{$\begin{array}{l}\text { Autoantibodies against } \\
\text { (at baseline if not stated otherwise) }\end{array}$} \\
\hline Double-stranded DNA & $38 \%$ & $43 \%$ & $36 \%$ & \\
\hline Double-stranded DNA (ever) & $61 \%$ & $64 \%$ & $60 \%$ & \\
\hline Cardiolipin lgG low titer & $48 \%$ & $50 \%$ & $47 \%$ & \\
\hline Cardiolipin lgG medium titer & $18 \%$ & $31 \%$ & $15 \%$ & 0.02 \\
\hline Cardiolipin IgM low titer & $16 \%$ & $19 \%$ & $15 \%$ & \\
\hline Cardiolipin IgM medium titer & $8 \%$ & $14 \%$ & $6 \%$ & \\
\hline beta 2 glykoprotein-1\# & $22 \%$ & $31 \%$ & $20 \%$ & \\
\hline Lupus anticoagulant & $23 \%$ & $31 \%$ & $21 \%$ & \\
\hline Any antiphospholipid low titer & $60 \%$ & $62 \%$ & $60 \%$ & \\
\hline Any antiphospholipid medium titer & $39 \%$ & $55 \%$ & $36 \%$ & 0.03 \\
\hline Sjogrens syndrome A & $41 \%$ & $21 \%$ & $46 \%$ & 0.003 \\
\hline Sjogrens syndrome B & $22 \%$ & $10 \%$ & $25 \%$ & \\
\hline Smith & $10 \%$ & $7 \%$ & $10 \%$ & \\
\hline Ribonucleoprotein & $19 \%$ & $21 \%$ & $19 \%$ & \\
\hline \multicolumn{5}{|l|}{ Medication } \\
\hline Steroids & $51 \%$ & $48 \%$ & $62 \%$ & \\
\hline Cyclofosfamid (ever use) & $17 \%$ & $26 \%$ & $16 \%$ & \\
\hline
\end{tabular}


Table 2 Baseline characteristics of patients with SLE $(n=208)$ (Continued)

\begin{tabular}{|c|c|c|c|c|}
\hline Azathioprine & $12 \%$ & $12 \%$ & $12 \%$ & \\
\hline Chloroquine/hydroxychloroquine & $27 \%$ & $14 \%$ & $30 \%$ & 0.04 \\
\hline Warfarin & $13 \%$ & $29 \%$ & $10 \%$ & 0.001 \\
\hline Acetylsalicylic acid & $21 \%$ & $33 \%$ & $18 \%$ & 0.02 \\
\hline Statins & $2 \%$ & $5 \%$ & $1 \%$ & 0.05 \\
\hline Methotrexate & $4 \%$ & $7 \%$ & $1 \%$ & \\
\hline Cyclosporine & $3 \%$ & $10 \%$ & $1 \%$ & 0.004 \\
\hline \multicolumn{5}{|l|}{ Inflammatory markers } \\
\hline High sensitivity $C$ reactive protein, $\mathrm{mg} / /^{*}$ \# & $2.2(0.8-6.2)$ & $5.1(2.2-12.1)$ & $1.8(0.7-4.5)$ & 0.0002 \\
\hline Fibrinogen, $g / /^{*} \#$ & $3.7(2.9-4.6)$ & $4.3(3.5-5.2)$ & $3.4(2.9-4.4)$ & $<0.0001$ \\
\hline$\alpha-1$ antitrypsine, g/l \# & $1.5(1.3-1.8)$ & $1.7(1.5-2.1)$ & $1.5(1.3-1.7)$ & 0.0001 \\
\hline Serum amyloid $\mathrm{A}, \mathrm{mg} / \mathrm{l}^{*} \#$ & $5.4(2.7-12)$ & $10.5(5.9-21.6)$ & $4.7(2.4-9.6)$ & \\
\hline Interleukin-6, ng/l* \# & $3.5(2.1-7.0)$ & $5.2(2.9-9.7)$ & $3.1(2.0-6.2)$ & \\
\hline Complement factor 3, g/l \# & $1.0(0.8-1.2)$ & $1.0(0.7-1.3)$ & $1.0(0.8-1.2)$ & \\
\hline Complement factor 3 degradation products, $\mathrm{mg} / /^{*} \#$ & $11.9(9.6-14.9)$ & $13.4(10.8-16.6)$ & $11.6(9.5-14.5)$ & 0.02 \\
\hline Complement factor $4\left(\mathrm{~g} / \mathrm{l}^{*} \#\right.$ & $0.2(0.1-0.2)$ & $0.2(0.1-0.2)$ & $0.2(0.1-0.2)$ & \\
\hline \multicolumn{5}{|l|}{ Endothelial markers } \\
\hline Soluble vascular cell adhesion molecule $1, \mathrm{ng} / /^{*}$ \# & $315(258-392)$ & $377(297-526)$ & $306(250-375)$ & $<0.0001$ \\
\hline Von Willebrand factor, \%*\# & $120(63-175)$ & $144(102-231)$ & $116(60-164)$ & 0.003 \\
\hline \multicolumn{5}{|l|}{ Markers of renal damage } \\
\hline Creatinine & $82(73-95)$ & $91(75-138)$ & $81(73-92)$ & $<0.0001$ \\
\hline Modification of Diet in Renal Disease formula, $\mathrm{ml} / \mathrm{min} / 1.73 \mathrm{~m} 2$ & $66.6(54.3-79.1)$ & $54.6(36.6-76.9)$ & $67.7(59.5-79.9)$ & 0.0005 \\
\hline Cystatin C GFR & $80(61-107)$ & $55(27-83)$ & $86(71-114)$ & $<0.0001$ \\
\hline Cystatin $C, \mathrm{mg} / \mathrm{l}^{*}$ & $1.0(0.8-1.2)$ & $1.3(1.0-2.2)$ & $0.9(0.8-1.1)$ & $<0.0001$ \\
\hline Blod urea nitrogen, $\mathrm{mmol} / /^{*} \#$ & $5.8(4.7-7.3)$ & $7.3(6.1-13.6)$ & $5.5(4.5-6.7)$ & \\
\hline Pathologic urine & $24 \%$ & $22 \%$ & $29 \%$ & \\
\hline \multicolumn{5}{|l|}{ Other biomarkers } \\
\hline Homocysteine, $\mathrm{mol} / /^{*} \#$ & $12.5(10.1-16.5)$ & $14.3(10.8-20.6)$ & $12(10.0-15.3)$ & 0.002 \\
\hline
\end{tabular}

Distributions are given as $\%$ or median (interquartile range), except for SCORE, where mean values are given. $P$-values $\leq 0.05$ are presented. ${ }^{*}$ Variables with nonnormal distribution. \# Analysis of frozen samples. §According to American College of Rheumatology (ACR) criteria [23]. १Positive antibody against cardiolipin IgG/ IgM at low and medium titer, respectively, beta ${ }_{2}$ glykoprotein-1, or a positive lupus anticoagulant test. Hypertension, systolic blood pressure $>140 \mathrm{~mm} \mathrm{Hg}$ and/or diastolic blood pressure $>90 \mathrm{mmHg}$ and/or treatment for hypertension; hypercholesterolemia, total cholesterol level > $5.2 \mathrm{mmol} / \mathrm{L}$; established arterial disease, history of myocardial infarction, angina pectoris, ischemic cerebrovascular disease, ischemic peripheral arterial disease at baseline; SCORE, systematic coronary risk evaluation calculated on 124 patients 40 to 65 years old; SLICC, systemic lupus international collaborating clinics; pathologic urine, as defined by the systemic lupus activity measure (SLAM). Data were missing as follows (deceased/survivors): low and high density lipoprotein (5/11), triglycerides (0/1), Apolipoprotein B/ Apolipoprotein A (1/0), leucopenia (1/0), thrombocytopenia (1/3), cyclophosphamide ever (15/3), statins (0/3), cyklosporin (0/1), $\alpha-1$ antitrypsine (0/1), serum amyloid (1/4), complement factor (C)3 (0/3), C3 degradation products (0/3), C 4 (0/3), soluble vascular cell adhesion molecule 1 (1/2), von Willebrand factor (1/2), cystatin C/cystatin C glomerular filtration rate (GFR) (1/0), urea (3/3), homocysteine (2/5).

$\mathrm{C}$ is strongly predictive of mortality and that it merits further evaluation as a biomarker in SLE.

sVCAM-1 was a strong risk factor for OM and in particular for CVM, underscoring the importance of endothelial activation for CVM in lupus. Endothelial biomarkers have not previously been investigated in the context of mortality in SLE. Levels of SVCAM-1 are elevated in SLE patients with manifest CVD [40] and together with vWf, another endothelial marker, they predicted the first arterial event [26]. An association with atherosclerosis has been demonstrated both in the general population and in lupus [4].

Systemic inflammation is associated with CVD, CVM and $\mathrm{N}-\mathrm{VM}$ in the general population $[41,42]$ and may be associated with CVD in lupus [26,43-45], although the impact on mortality has not yet been well studied.
We demonstrated that several inflammatory markers were associated with all-cause mortality. For CVM, hsCRP (and $\alpha 1$-antitrypsin among women) had the greatest impact.

$\mathrm{a} \beta_{2} \mathrm{GP} 1$ and any aPL at medium titer were associated with CVM in multivariable analyses. This observation is in accordance with previous studies, where aPLs were associated with cardiovascular events $[26,44]$. The high prevalence of aCL in our study is probably due to a low cutoff for positivity at our laboratory in the mid 90's, when baseline data were collected. Since then, much work has been carried out to evaluate more appropriate cutoffs for aCL in relation to what is clinically significant [46]. When we used a cutoff at medium titer, the prevalence of aCL was more in accordance with other studies [47]. Furthermore, medium titer of aPLs were 
Table 3 Age-adjusted Cox regression analysis

\begin{tabular}{|c|c|c|c|c|c|c|}
\hline & $\begin{array}{c}\mathrm{OM} \\
\mathrm{n}=42\end{array}$ & & $C V M n=20$ & & $\begin{array}{c}\mathrm{N}-\mathrm{VM} \\
\mathrm{n}=18\end{array}$ & \\
\hline & $P$-value & $\begin{array}{c}\mathrm{HR} \\
(95 \% \mathrm{Cl})\end{array}$ & $P$-value & $\begin{array}{c}\mathrm{HR} \\
(95 \% \mathrm{Cl})\end{array}$ & $P$-value & $\begin{array}{c}\text { HR } \\
(95 \% \mathrm{Cl})\end{array}$ \\
\hline Age univariate & $<0.0001$ & $\begin{array}{c}1.1 \\
(1.0-1.1)\end{array}$ & $<0.0001$ & $\begin{array}{c}1.07 \\
(1.04-1.1)\end{array}$ & 0.02 & $\begin{array}{c}1,04 \\
(1.00-1.1)\end{array}$ \\
\hline
\end{tabular}

Age adjusted

Traditional risk factors

\begin{tabular}{|c|c|c|c|c|c|c|}
\hline Smoking & & & 0.02 & $3.3(1.3-8.6)$ & & \\
\hline Hypertension & 0.02 & $\begin{array}{c}2.2 \\
(1.1-4.5)\end{array}$ & & & 0.04 & $2.8(1.0-7.9)$ \\
\hline \multicolumn{7}{|l|}{ Cholesterol, mmol/l } \\
\hline Triglycerides, mmol/l† & 0.02 & $\begin{array}{c}1.9 \\
(1.1-3.3)\end{array}$ & & & 0.04 & $2.5(1.1-5.7)$ \\
\hline Established arterial disease & $<0.0001$ & $\begin{array}{c}4.7 \\
(2.4-9.1)\end{array}$ & 0.0006 & $\begin{array}{c}5.4 \\
(2.1-13.6)\end{array}$ & 0.0006 & $\begin{array}{c}6.3 \\
(2.3-16.9)\end{array}$ \\
\hline \multicolumn{7}{|l|}{ Lupus manifestations } \\
\hline Nephritis & & & 0.03 & $3.0(1.2-7.9)$ & & \\
\hline Neurological disorder & & & 0.04 & $2.9(1.1-7.3)$ & & \\
\hline Epilepsy & 0.03 & $\begin{array}{c}2.3 \\
(1.1-4.5)\end{array}$ & 0.01 & $3.7(1.4-9.3)$ & & \\
\hline SLICC > $1[25]$ & $<0.0001$ & $\begin{array}{c}6.3 \\
(2.2-26.5)\end{array}$ & 0.04 & $\begin{array}{c}3.7 \\
(1.0-23.5)\end{array}$ & 0.002 & $\begin{array}{c}9.6 \\
(1.9-181.1)\end{array}$ \\
\hline SLAM > 6 [24] & 0.02 & $\begin{array}{c}2.1 \\
(1.1-4.5)\end{array}$ & & & & \\
\hline \multicolumn{7}{|l|}{ Autoantibodies against } \\
\hline Double-stranded DNA & 0.05 & $\begin{array}{c}1.9 \\
(1.0-3.6)\end{array}$ & & & 0.02 & $3.1(1.2-8.3)$ \\
\hline Cardiolipin IgG low titer & & & & & 0.03 & $2.9(1.1-8.1)$ \\
\hline Cardiolipin IgG medium titer & 0.006 & $\begin{array}{c}2.7 \\
(1.4-5.3)\end{array}$ & 0.04 & $3.2(1.1-8.5)$ & 0.02 & $3.6(1.3-9.6)$ \\
\hline beta $_{2}$ glykoprotein-1 & & & 0.04 & $2.9(1.0-7.8)$ & & \\
\hline Any aPL medium titer 9 & 0.02 & $\begin{array}{c}2.1 \\
(1.1-3.9)\end{array}$ & 0.03 & $2.8(1.1-7.7)$ & 0.03 & $2.8(1.1-7.6)$ \\
\hline Sjogrens syndrome A & 0.01 & $\begin{array}{c}0.4 \\
(0.2-0.8)\end{array}$ & & & & \\
\hline Sjogrens syndrome B & 0.04 & $\begin{array}{c}0.4 \\
(0.1-0.9)\end{array}$ & & & 0.003 & $\begin{array}{c}5 \times 10^{-7} \\
(0-0.4)\end{array}$ \\
\hline \multicolumn{7}{|l|}{ Medications } \\
\hline Warfarin & 0.004 & $\begin{array}{c}2.9 \\
(1.4-5.7)\end{array}$ & 0.01 & $\begin{array}{c}4.1 \\
(1.4-11.1)\end{array}$ & 0.03 & $3.3(1.1-8.3)$ \\
\hline Hyperlipidemia & & & 0.03 & $\begin{array}{c}7.8 \\
(1.2-27.9)\end{array}$ & & \\
\hline \multicolumn{7}{|l|}{ Inflammatory markers } \\
\hline High sensitivity CRP, mg/l† & 0.01 & $\begin{array}{c}1.3 \\
(1.0-1.7)\end{array}$ & 0.02 & $\begin{array}{c}1.5 \\
(1.1-2.1)\end{array}$ & & \\
\hline Fibrinogen, g/l† & 0.0002 & $9.5(3.0-31)$ & 0.02 & $\begin{array}{c}10.2 \\
(1.6-70)\end{array}$ & 0.0005 & $19.9(3.8-110)$ \\
\hline$\alpha-1$ antitrypsin, g/l & 0.002 & $\begin{array}{c}3.1 \\
(1.5-5.8)\end{array}$ & & & 0.001 & $\begin{array}{c}5.1 \\
(2.0-12.0)\end{array}$ \\
\hline Serum amyloid A, mg/l† & 0.01 & $\begin{array}{c}1.4 \\
(1.1-1.8)\end{array}$ & 0.03 & $1.5(1.0-2.1)$ & & \\
\hline \multicolumn{7}{|l|}{ Endothelial markers } \\
\hline Soluble vascular cell adhesion molecule, ng/l† & 0.0005 & $\begin{array}{c}4.0 \\
(1.8-8.2)\end{array}$ & 0.004 & $\begin{array}{c}4.9 \\
(1.7-13.5)\end{array}$ & & \\
\hline Von Willebrand factor, \%† & 0.009 & $\begin{array}{c}1.9 \\
(1.2-3.0)\end{array}$ & & & & \\
\hline
\end{tabular}


Table 3 Age-adjusted Cox regression analysis (Continued)

\begin{tabular}{|c|c|c|c|c|c|c|}
\hline \multicolumn{7}{|c|}{ Markers of renal damage } \\
\hline Cystatin C GFR & $<0.0001$ & $\begin{array}{c}0.4 \\
(0.2-0.5)\end{array}$ & 0.001 & $0.3(0.2-0.6)$ & 0.0002 & $0.3(0.2-0.5)$ \\
\hline Cystatin C† & $<0.0001$ & $\begin{array}{c}4.4 \\
(2.4-7.9)\end{array}$ & 0.0009 & $5.3(2.0-13)$ & 0.0002 & $\begin{array}{c}5.7 \\
(2.4-12.8)\end{array}$ \\
\hline Creatinine & 0.003 & $\begin{array}{c}1.1 \\
(1.0-1.1)\end{array}$ & 0.02 & $\begin{array}{c}4.1 \\
(1.3-10.2)\end{array}$ & 0.003 & $4.3(1.7-9.2)$ \\
\hline Urea, mmol/l† & 0.01 & $2.1(1.2-3.2)$ & & & 0.02 & $2.3(1.1-4.0)$ \\
\hline Pathologic urine & 0.04 & $2.2(1.0-4.4)$ & & & 0.04 & $3.5(1.1-8.1)$ \\
\hline
\end{tabular}

Only variables with $P$-values $\leq 0.05$ in either group are presented. Calculations were done using age-adjusted Cox regression. $+C a l c u l a t i o n s$ on log transformed values. Any aPLף, any antiphospholipid antibody, positive antibody against cardiolipin lgG/lgM at medium titer, beta 2 glykoprotein-1 or a positive lupus anticoagulant test. Hypertension, systolic blood pressure $>140 \mathrm{~mm} \mathrm{Hg}$ and/or diastolic blood pressure $>90 \mathrm{mmHg}$ and/or treatment for hypertension; hypercholesterolemia, total cholesterol level > $5.2 \mathrm{mmol} / \mathrm{L}$; established arterial disease, history of myocardial infarction, angina pectoris, ischemic cerebrovascular disease, ischemic peripheral arterial disease at baseline; SCORE, systematic coronary risk evaluation calculated on 124 patients 40 to 65 years old; SLICC, systemic lupus international collaborating clinics; pathologic urine, as defined by the systemic lupus activity measure (SLAM); OM, overall mortality; CVM, cardiovascular mortality; N-VM, non-vascular mortality. HR, hazard ratio

predictive of CVM, indicating that these are more clinically relevant levels. However, we have previously demonstrated that aPL only at a low cutoff were predictive of the first arterial event [26]. $a \beta_{2} \mathrm{GP} 1$ was analyzed later on frozen samples, with the method currently used at our immunological laboratory. Taken together, our results demonstrate that clinically relevant levels for aPL need to be further studied and standardized. Studies in our research group are ongoing to investigate these issues.

SSA/SSB antibodies often occur together with skin manifestations in SLE. We noted an inverse association between positivity for SSB antibodies and N-VM. SSA antibodies [20] and photosensitivity [10] were previously inversely linked to mortality. Together these results indicate that lupus patients with SSA/SSB positivity and/or skin manifestations have a better prognosis, further illustrating that sub-phenotypes of SLE have differentiated risk profiles [26].
Smoking was the only traditional CVD risk factor associated with CVM in multivariable analyses. Smoking has previously been shown to predict cardiovascular events in SLE $[26,44]$. Hypertension was predictive of mortality in earlier SLE studies $[19,48]$. As definitions differ and antihypertensive agents are used in the treatment of nephritis, even in the absence of high blood pressure, it is nowadays difficult to assess its contribution to mortality. Hyperlipidemia was not an important risk factor for mortality in multivariable analyses.

SCORE is a widespread clinical cardiovascular risk scoring system distributed through the European Society of Cardiology. SCORE underestimated CVM among our SLE patients (nine observed vs. four predicted cases), but the difference was not significant. This is nevertheless interesting as it suggests that optimal preventive cardiovascular strategies in lupus need to target other factors in addition to traditional CVD risk factors.

Table 4 Multivariable Cox regression model adjusted for age, arterial disease and cystatin C (208 patients).

\begin{tabular}{|c|c|c|c|c|c|c|}
\hline & OM & & CVM & & N-VM & \\
\hline & $P$-value & $\mathrm{HR}(95 \% \mathrm{Cl})$ & P-value & $\mathrm{HR}(95 \% \mathrm{Cl})$ & $P$-value & $\mathrm{HR}(95 \% \mathrm{Cl})$ \\
\hline Smoking & & & 0.02 & $3.4(1.3-9.2)$ & & \\
\hline SLICC $>1[25]$ & 0.008 & $4.1(1.4-17.3)$ & & & 0.05 & $5.6(1.0-103.6)$ \\
\hline $\mathrm{a} \beta_{2} \mathrm{GP} 1$ & & & 0.03 & $3.4(1.2-9.7)$ & & \\
\hline Any aPL medium titer & & & 0.05 & $2.8(1.0-8.2)$ & & \\
\hline Sjogrens syndrome B antibodies & & & & & 0.02 & $1.3 e-6(0-0.7)$ \\
\hline Warfarin & & & 0.05 & $3.4(1.0-10.4)$ & & \\
\hline High sensitivity CRP & 0.04 & $1.3(1.0-1.6)$ & 0.02 & $1.6(1.1-2.3)$ & & \\
\hline Fibrinogen & 0.04 & $3.7(1.0-13.1)$ & & & 0.05 & $6.7(1.0-45.4)$ \\
\hline$\alpha$-1-antitrypsine & 0.007 & $2.7(1.3-5.2)$ & & & 0.004 & $4.3(1.6-10.7)$ \\
\hline Soluble vascular cell adhesion molecule 1 & 0.05 & $2.7(1.0-6.7)$ & 0.02 & $5.3(1.3-19.3)$ & & \\
\hline
\end{tabular}

$\mathrm{a} \beta_{2} \mathrm{GP1}$, anti- $\beta_{2}$ glycoprotein-1; any aPL, any antiphospholipid; positive antibody against cardiolipin IgG/lgM at medium titer, $\beta_{2} \mathrm{GP1}$ or a positive lupus anticoagulant test; OM, overall mortality; CVM, cardiovascular mortality; HR, hazard ratio; N-VM, non-vascular mortality; SLICC, systemic lupus international collaborating clinics. Age, arterial disease and cystatin $\mathrm{C}$ were included in all multivariable models. Each variable that was significant in age-adjusted Cox regression (Table 3) for OM, CVM and N-VM, was included one by one, together with the three above-mentioned variables, creating a model with four variables. The table presents the results of the significant associations. Only variables that have $P$-values $\leq 0.05$ in either group are presented. In this way, five different multivariable models are presented for OM, six for CVM, and four for N-VM, respectively. 
Table 5 Multivariable Cox regression model adjusted for age, arterial disease and cystatin C

\begin{tabular}{|c|c|c|c|c|c|c|}
\hline & OM & & CVM & & $\mathrm{N}-\mathrm{VM}$ & \\
\hline & $P$-value & $\mathrm{HR}(95 \% \mathrm{Cl})$ & $P$-value & $\mathrm{HR}(95 \% \mathrm{Cl})$ & $P$-value & $\operatorname{HR}(95 \% \mathrm{Cl})$ \\
\hline Smoking & & & 0.02 & $3.6(1.3-10.4)$ & & \\
\hline SLICC >1 $[25]$ & 0.01 & $3.8(1.3-16.4)$ & & & & \\
\hline$a \beta_{2} \mathrm{GP} 1$ & & & 0.02 & $3.6(1.2-10.6)$ & & \\
\hline Sjogrens syndrome B antibodies & & & & & 0.02 & $9.1 \mathrm{e}-7(0-0.8)$ \\
\hline Warfarin & & & 0.04 & $3.7(1.1-11.9)$ & & \\
\hline High sensitivity CRP & & & 0.01 & $1.6(1.1-2.4)$ & & \\
\hline \multicolumn{7}{|l|}{ Fibrinogen } \\
\hline$\alpha$-1-antitrypsine & 0.02 & $3.0(1.4-6.4)$ & 0.04 & $3.3(1.1-9.2)$ & 0.02 & $4.3(1.4-12.7)$ \\
\hline Soluble vascular cell adhesion molecule 1 & & & 0.02 & $5.2(1.3-18.8)$ & & \\
\hline
\end{tabular}

$\mathrm{a} \beta_{2} \mathrm{GP1}$, anti- $\beta_{2}$ glycoprotein-1; SLICC, systemic lupus international collaborating clinics; CRP, C-reactive protein; HR, hazard ratio; OM, overall mortality; CVM, cardiovascular mortality; N-VM, non-vascular mortality. Only $P$-values $\leq 0.05$ are presented.

Notably, four SLE patients (10\%) in this cohort died from PHT. They died at a young mean age (41 years). Death from PHT was $15 \%$ in a Korean cohort [49], but in most studies PHT is not reported as a prominent cause of death.

Detailed baseline information and complete follow-up are strengths of this study. Assigning a principle cause of death is difficult, particularly in patients with chronic diseases with numerous co-morbidities. We did not rely on death certificates only, but supplemented these data with autopsy protocols and medical charts, thus using all available sources to determine a main cause of death. Because national mortality data are based solely on international classification of diseases (ICD) codes, derived from only one of the data sources we considered in the determination of cause of death, cause-specific SMRs could not be calculated. It is difficult to compare causes of death in our cohort and in the general population for the same reason.

The majority of our patients were female, and of European Caucasian origin. Because Swedish healthcare is tax-funded, granting universal access, patients with lower socioeconomic status have the same access with the same threshold maximum payment per year. Therefore further studies in male patients, other ethnic cohorts and socioeconomic groups are needed. Furthermore, we had limited statistical power. For example, only 13 deaths occurred among patients aged 50 years or younger, prohibiting the evaluation of effect modification by age. Another limitation of this study is the assessment of risk factors at baseline only, which makes it difficult to evaluate development of disease manifestations and other events during followup. Finally, we did not adjust for multiple comparisons, and therefore $P$-values close to 0.05 should be interpreted with caution.

\section{Conclusions}

This study demonstrates that high levels of cystatin C strongly predicted all cause mortality. Additionally, CVM was associated with high levels of sVCAM-1,
hsCRP and aPL, demonstrating that systemic and vascular inflammation, and prothrombotic autoantibodies are important risk factors for CVM. With the exception of smoking, traditional risk factors had less impact. Thus, new biomarkers differentiate SLE patients with favorable vs. more severe prognosis.

\section{Abbreviations}

a $\beta_{2}$ GP1: anti- $\beta_{2}$ glycoprotein-1; aCL: anticardiolipin antibodies; AIC: akaike information criterion; ANA: antinuclear antibodies; ANOVA: analysis of variance; aPL: antiphospholipid antibody; CVD: cardiovascular disease; CVM: cardiovascular mortality; eGFR: estimated glomerular filtration rate; ELISA: enzyme-linked immunosorbent assays; HDL: high-density lipoprotein; HRP: horseradish peroxidase; hsCRP: high sensitivity C-reactive protein; IL-6: interleukin 6; LAC: lupus anticoagulant; LDL: low-density lipoprotein; MDRD: modification of diet in renal disease formula; N-VM: non-vascular mortality; OM: overall mortality; PHT: pulmonary hypertension; RNP: ribonucleoprotein SAA: serum amyloid A; SCORE: systematic coronary risk evaluation; SLAM: systemic lupus activity measure; SLE: systemic lupus erythematosus; SLICC: systemic lupus international collaborating clinics; Sm: smith antigen; SMR: standardized mortality ratio; SSA/SSB: sjogrens syndrome A/B; SVCAM-1: soluble vascular cell adhesion molecule-1; vWf: von willebrand factor.

\section{Acknowledgements}

We are grateful to Jill Gustafsson, Sonia Möller, Susanne Pettersson and Eva Jemseby for management of patient cohorts and blood sampling. This work was supported by the Swedish Heart-Lung Foundation, Stockholm County Council and Karolinska Institutet (ALF), The King Gustaf V 80th Birthday Fund The Swedish Rheumatism Association, The Åke Wiberg Foundation, Alex and Eva Wallströms Foundation, Karolinska Institutet's Foundations, and The Foundation in memory of Clas Groschinsky, The Swedish Society of Medicine.

\section{Author details}

'Rheumatology Unit, Department of Medicine, Karolinska University Hospital, Solna, Karolinska Institutet, SE-171 76 Stockholm, Sweden. ${ }^{2}$ Clinical Epidemiology Unit, Department of Medicine, Karolinska Institutet, SE-171 76 Stockholm, Sweden. ${ }^{3}$ Unit of Clinical Immunology, Department of Clinical Immunology and Transfusion Medicine, Karolinska University Hospital, Solna, Karolinska Institutet, SE-171 76 Stockholm, Sweden. ${ }^{4}$ Department of Laboratory Medicine, Karolinska University Hospital, Karolinska Institutet, SE17176 Stockholm, Sweden. ${ }^{5}$ Department of Clinical Chemistry and Pharmacology, Akademiska Hospital, SE-751 85 Uppsala, Sweden.

\section{Authors' contributions}

$J G$ acquired, analyzed and interpreted the data and drafted the manuscript JFS analyzed the data and drafted the manuscript. KE coordinated and acquired the analysis of autoantibodies. IEL acquired the data. $\mathrm{LOH}$ and $\mathrm{AL}$ 
coordinated and acquired the laboratory data. ES conceived and designed the study, acquired, analyzed and interpreted the data and drafted the manuscript. All authors reviewed and approved the final manuscript.

\section{Competing interests}

The authors declare that they have no competing interests.

Received: 25 September 2011 Revised: 23 January 2012

Accepted: 5 March 2012 Published: 5 March 2012

\section{References}

1. Ahmad Y, Shelmerdine J, Bodill H, Lunt M, Pattrick MG, Teh LS, Bernstein RM, Walker MG, Bruce IN: Subclinical atherosclerosis in systemic lupus erythematosus (SLE): the relative contribution of classic risk factors and the lupus phenotype. Rheumatology (Oxford) 2007, 46:983-988.

2. Manzi S, Selzer F, Sutton-Tyrrell K, Fitzgerald SG, Rairie JE, Tracy RP, Kuller LH: Prevalence and risk factors of carotid plaque in women with systemic lupus erythematosus. Arthritis Rheum 1999, 42:51-60.

3. Roman MJ, Shanker BA, Davis A, Lockshin MD, Sammaritano L, Simantov R, Crow MK, Schwartz JE, Paget SA, Devereux RB, Salmon JE: Prevalence and correlates of accelerated atherosclerosis in systemic lupus erythematosus. N Engl J Med 2003, 349:2399-2406.

4. de Leeuw K, Freire B, Smit AJ, Bootsma H, Kallenberg CG, Bijl M: Traditional and non-traditional risk factors contribute to the development of accelerated atherosclerosis in patients with systemic lupus erythematosus. Lupus 2006, 15:675-682.

5. Merrell M, Shulman LE: Determination of prognosis in chronic disease, illustrated by systemic lupus erythematosus. J Chronic Dis 1955, 1:12-32

6. Kasitanon N, Magder LS, Petri M: Predictors of survival in systemic lupus erythematosus. Medicine (Baltimore) 2006, 85:147-156.

7. Funauchi M, Shimadzu H, Tamaki C, Yamagata T, Nozaki Y, Sugiyama M, Ikoma S, Kinoshita K: Survival study by organ disorders in 306 Japanese patients with systemic lupus erythematosus: results from a single center. Rheumatol Int 2007, 27:243-249.

8. Urowitz MB, Gladman DD, Tom BD, Ibanez D, Farewell VT: Changing patterns in mortality and disease outcomes for patients with systemic lupus erythematosus. J Rheumatol 2008, 35:2152-2158.

9. Mok CC, Kwok CL, Ho LY, Chan PT, Yip SF: Life expectancy, standardized mortality ratios, and causes of death in six rheumatic diseases in Hong Kong, China. Arthritis Rheum 2011, 63:1182-1189.

10. Jacobsen $S$, Petersen J, Ullman S, Junker P, Voss A, Rasmussen JM, Tarp U, Poulsen LH, van Overeem Hansen G, Skaarup B, Hansen TM, Podenphant J, Halberg P: A multicentre study of 513 Danish patients with systemic lupus erythematosus. II. Disease mortality and clinical factors of prognostic value. Clin Rheumatol 1998, 17:478-484.

11. Chambers SA, Allen E, Rahman A, Isenberg D: Damage and mortality in a group of British patients with systemic lupus erythematosus followed up for over 10 years. Rheumatology (Oxford) 2009, 48:673-675.

12. Rabbani MA, Habib HB, Islam M, Ahmad B, Majid S, Saeed W, Shah SM, Ahmad A: Survival analysis and prognostic indicators of systemic lupus erythematosus in Pakistani patients. Lupus 2009, 18:848-855.

13. Wadee S, Tikly M, Hopley M: Causes and predictors of death in South Africans with systemic lupus erythematosus. Rheumatology (Oxford) 2007, 46:1487-1491.

14. Bjornadal L, Yin L, Granath F, Klareskog L, Ekbom A: Cardiovascular disease a hazard despite improved prognosis in patients with systemic lupus erythematosus: results from a Swedish population based study 1964-95. J Rheumatol 2004, 31:713-719.

15. Bernatsky S, Boivin JF, Joseph L, Manzi S, Ginzler E, Gladman DD, Urowitz M, Fortin PR, Petri M, Barr S, Gordon C, Bae SC, Isenberg D, Zoma A, Aranow C, Dooley MA, Nived O, Sturfelt G, Steinsson K, Alarcon G, Senecal JL, Zummer M, Hanly J, Ensworth S, Pope J, Edworthy S, Rahman A, Sibley J, ElGabalawy H, McCarthy T, et al: Mortality in systemic lupus erythematosus. Arthritis Rheum 2006, 54:2550-2557.

16. Moss KE, loannou Y, Sultan SM, Haq I, Isenberg DA: Outcome of a cohort of 300 patients with systemic lupus erythematosus attending a dedicated clinic for over two decades. Ann Rheum Dis 2002, 61:409-413.

17. Stahl-Hallengren C, Jonsen A, Nived O, Sturfelt G: Incidence studies of systemic lupus erythematosus in Southern Sweden: increasing age, decreasing frequency of renal manifestations and good prognosis. J Rheumatol 2000, 27:685-691.
18. Reveille JD, Bartolucci A, Alarcon GS: Prognosis in systemic lupus erythematosus. Negative impact of increasing age at onset, black race, and thrombocytopenia, as well as causes of death. Arthritis Rheum 1990, 33:37-48.

19. Abu-Shakra M, Urowitz MB, Gladman DD, Gough J: Mortality studies in systemic lupus erythematosus. Results from a single center. II. Predictor variables for mortality. J Rheumatol 1995, 22:1265-1270.

20. Manger K, Manger B, Repp R, Geisselbrecht M, Geiger A, Pfahlberg A, Harrer T, Kalden JR: Definition of risk factors for death, end stage renal disease, and thromboembolic events in a monocentric cohort of 338 patients with systemic lupus erythematosus. Ann Rheum Dis 2002, 61:1065-1070.

21. Doria A, laccarino L, Ghirardello A, Zampieri S, Arienti S, Sarzi-Puttini P, Atzeni F, Piccoli A, Todesco S: Long-term prognosis and causes of death in systemic lupus erythematosus. Am J Med 2006, 119:700-706.

22. Conroy RM, Pyorala K, Fitzgerald AP, Sans S, Menotti A, De Backer G, De Bacquer D, Ducimetiere P, Jousilahti P, Keil U, Njolstad I, Oganov RG, Thomsen T, Tunstall-Pedoe H, Tverval A, Wedel H, Whincup P, Wilhelmsen L, Graham IM: Estimation of ten-year risk of fatal cardiovascular disease in Europe: the SCORE project. Eur Heart J 2003, 24:987-1003.

23. Tan EM, Cohen AS, Fries JF, Masi AT, McShane DJ, Rothfield NF, Schaller JG, Talal N, Winchester RJ: The 1982 revised criteria for the classification of systemic lupus erythematosus. Arthritis Rheum 1982, 25:1271-1277.

24. Liang MH, Socher SA, Roberts WN, Esdaile JM: Measurement of systemic lupus erythematosus activity in clinical research. Arthritis Rheum 1988, 31:817-825.

25. Gladman D, Ginzler E, Goldsmith C, Fortin P, Liang M, Urowitz M, Bacon P, Bombardieri S, Hanly J, Hay E, Isenberg D, Jones J, Kalunian K, Maddison P, Nived O, Petri M, Richter M, Sanchez-Guerrero J, Snaith M, Sturfelt G, Symmons D, Zoma A: The development and initial validation of the Systemic Lupus International Collaborating Clinics/American College of Rheumatology damage index for systemic lupus erythematosus. Arthritis Rheum 1996, 39:363-369.

26. Gustafsson J, Gunnarsson I, Borjesson O, Pettersson S, Moller S, Fei GZ, Elvin K, Simard JF, Hansson LO, Lundberg IE, Larsson A, Svenungsson E: Predictors of the first cardiovascular event in patients with systemic lupus erythematosus - a prospective cohort study. Arthritis Res Ther 2009, 11:R186.

27. Heartscore. [http://www.heartscore.org].

28. Jacobsen S, Petersen J, Ullman S, Junker P, Voss A, Rasmussen JM, Tarp U, Poulsen LH, van Overeem Hansen G, Skaarup B, Hansen TM, Podenphant J, Halberg P: Mortality and causes of death of 513 Danish patients with systemic lupus erythematosus. Scand I Rheumatol 1999, 28:75-80.

29. Cervera R, Khamashta MA, Font J, Sebastiani GD, Gil A, Lavilla P, Mejia JC, Aydintug AO, Chwalinska-Sadowska H, de Ramon E, Fernandez-Nebro A, Galeazzi M, Valen M, Mathieu A, Houssiau F, Caro N, Alba P, RamosCasals $M$, Ingelmo $M$, Hughes GR: Morbidity and mortality in systemic lupus erythematosus during a 10-year period: a comparison of early and late manifestations in a cohort of 1,000 patients. Medicine (Baltimore) 2003, 82:299-308.

30. Nived O, Jonsen A, Bengtsson AA, Bengtsson C, Sturfelt G: High predictive value of the Systemic Lupus International Collaborating Clinics/American College of Rheumatology damage index for survival in systemic lupus erythematosus. J Rheumatol 2002, 29:1398-1400.

31. Shlipak MG, Weekley CC, Li Y, Hansson LO, Larsson A, Whooley M: Comparison of cardiovascular prognosis by 3 serum cystatin $\mathrm{C}$ methods in the heart and soul study. Clin Chem 2011, 57:737-745.

32. Levey AS, Bosch JP, Lewis JB, Greene T, Rogers N, Roth D: A more accurate method to estimate glomerular filtration rate from serum creatinine: a new prediction equation. Modification of Diet in Renal Disease Study Group. Ann Intern Med 1999, 130:461-470.

33. Faurschou M, Dreyer L, Kamper AL, Starklint H, Jacobsen S: Long-term mortality and renal outcome in a cohort of 100 patients with lupus nephritis. Arthritis Care Res (Hoboken) 2010, 62:873-880.

34. Pucci L, Triscornia S, Lucchesi D, Fotino C, Pellegrini G, Pardini E, Miccoli R, Del Prato S, Penno G: Cystatin C and estimates of renal function: searching for a better measure of kidney function in diabetic patients. Clin Chem 2007, 53:480-488.

35. Hsu CY, Chertow GM, Curhan GC: Methodological issues in studying the epidemiology of mild to moderate chronic renal insufficiency. Kidney Int 2002, 61:1567-1576. 
36. Risch L, Herklotz R, Blumberg A, Huber AR: Effects of glucocorticoid immunosuppression on serum cystatin $C$ concentrations in renal transplant patients. Clin Chem 2001, 47:2055-2059.

37. Keller CR, Odden MC, Fried LF, Newman AB, Angleman S, Green CA, Cummings SR, Harris TB, Shlipak MG: Kidney function and markers of inflammation in elderly persons without chronic kidney disease: the health, aging, and body composition study. Kidney Int 2007, 71:239-244.

38. Lee M, Saver JL, Huang WH, Chow J, Chang KH, Ovbiagele B: Impact of elevated cystatin C level on cardiovascular disease risk in predominantly high cardiovascular risk populations: a meta-analysis. Circ Cardiovasc Qual Outcomes 2010, 3:675-683.

39. Wu CK, Lin JW, Caffrey JL, Chang MH, Hwang JJ, Lin YS: Cystatin C and long-term mortality among subjects with normal creatinine-based estimated glomerular filtration rates: NHANES III (Third National Health and Nutrition Examination Survey). J Am Coll Cardiol 2010, 56:1930-1936.

40. Svenungsson E, Cederholm A, Jensen-Urstad K, Fei GZ, de Faire U, Frostegard J: Endothelial function and markers of endothelial activation in relation to cardiovascular disease in systemic lupus erythematosus. Scand J Rheumatol 2008, 37:352-359.

41. Kaptoge S, Di Angelantonio E, Lowe G, Pepys MB, Thompson SG, Collins R, Danesh J: C-reactive protein concentration and risk of coronary heart disease, stroke, and mortality: an individual participant meta-analysis. Lancet 2010, 375:132-140.

42. Danesh J, Lewington S, Thompson SG, Lowe GD, Collins R, Kostis JB, Wilson AC, Folsom AR, Wu K, Benderly M, Goldbourt U, Willeit J, Kiechl S, Yarnell JW, Sweetnam PM, Elwood PC, Cushman M, Psaty BM, Tracy RP, Tygbjaerg-Hansen A, Haverkate F, de Maat MP, Fowkes FG, Lee AJ, Smith FB, Salomaa V, Harald K, Rasi R, Vahtera E, Jousilahti P, et al: Plasma fibrinogen level and the risk of major cardiovascular diseases and nonvascular mortality: an individual participant meta-analysis. Jama 2005, 294:1799-1809.

43. Kao AH, Wasko MC, Krishnaswami S, Wagner J, Edmundowicz D, Shaw P, Cunningham AL, Danchenko N, Sutton-Tyrrell K, Tracy RP, Kuller LH, Manzi S: C-reactive protein and coronary artery calcium in asymptomatic women with systemic lupus erythematosus or rheumatoid arthritis. $\mathrm{Am} J$ Cardiol 2008, 102:755-760.

44. Toloza SM, Uribe AG, McGwin G Jr, Alarcon GS, Fessler BJ, Bastian HM, Vila LM, Wu R, Shoenfeld Y, Roseman JM, Reveille JD: Systemic lupus erythematosus in a multiethnic US cohort (LUMINA). XXIII. Baseline predictors of vascular events. Arthritis Rheum 2004, 50:3947-3957.

45. Svenungsson E, Jensen-Urstad K, Heimburger M, Silveira A, Hamsten A, de Faire U, Witztum JL, Frostegard J: Risk factors for cardiovascular disease in systemic lupus erythematosus. Circulation 2001, 104:1887-1893.

46. Miyakis S, Lockshin MD, Atsumi T, Branch DW, Brey RL, Cervera R, Derksen RH, PG DEG, Koike T, Meroni PL, Reber G, Shoenfeld Y, Tincani A, Vlachoyiannopoulos PG, Krilis SA: International consensus statement on an update of the classification criteria for definite antiphospholipid syndrome (APS). J Thromb Haemost 2006, 4:295-306.

47. Petri M: Update on anti-phospholipid antibodies in SLE: the Hopkins' Lupus Cohort. Lupus 2010, 19:419-423.

48. Seleznick MJ, Fries JF: Variables associated with decreased survival in systemic lupus erythematosus. Semin Arthritis Rheum 1991, 21:73-80.

49. Kim WU, Min JK, Lee SH, Park SH, Cho CS, Kim HY: Causes of death in Korean patients with systemic lupus erythematosus: a single center retrospective study. Clin Exp Rheumatol 1999, 17:539-545.

doi:10.1186/ar3759

Cite this article as: Gustafsson et al:: Risk factors for cardiovascular mortality in patients with systemic lupus erythematosus, a prospective cohort study. Arthritis Research \& Therapy 2012 14:R46.

\section{Submit your next manuscript to BioMed Central and take full advantage of:}

- Convenient online submission

- Thorough peer review

- No space constraints or color figure charges

- Immediate publication on acceptance

- Inclusion in PubMed, CAS, Scopus and Google Scholar

- Research which is freely available for redistribution

Submit your manuscript at www.biomedcentral.com/submit 